\title{
Stem Cell Research and Regenerative Medicine
}

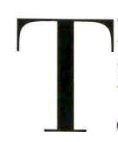

The ability to change one cell type into another has far-reaching implications for both basic and clinical research.

In 2009, led by ZHOU Qi of the CAS Institute of Zoology (IOZ) and ZENG Fanyi of Shanghai Jiao Tong University, Chinese scientists created the first viable mouse from induced stem (iPS) cells (Nature 2009, doi: 10.1038/nature0826?). This feat gave an answer to the question whether iPS cells can attain true pluripotency similar to embryonic stem (ES) cells. Before this progress, viable mice could only be produced via ES cells. The establishment of iPS technology, winning the 2013 Outstanding Scientific and Technological Achievement Award of the Chinese Academy of Sciences. also provides a technical platform for apply iPS in the fields of stem cells, developmental biology and regenerative medicine.

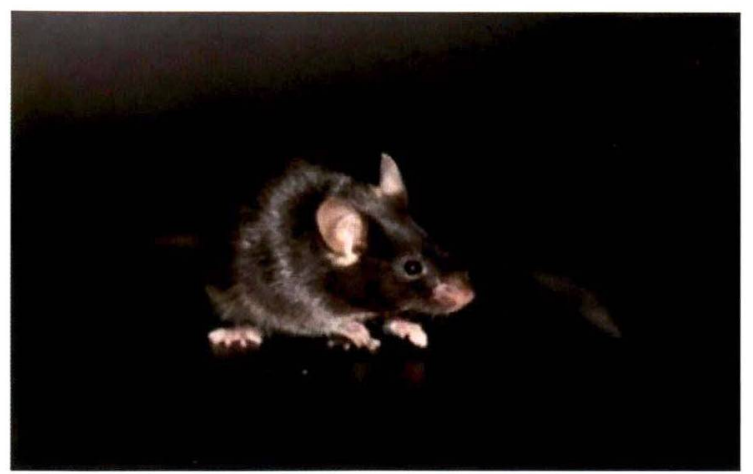

The first iPS-cell mouse named Xiaoxiao was created in a Chinese lab by CAS scientists. (Credit: IOZ)

The first iPS mouse was named Xiaoxiao (literately Tinv). in the hope that a small step of this tiny mouse could move the field of stem cell research forward. "Most research progresses in small steps. Xiaoxiao represents a jump forward." said Bruce Whitelaw, head of the Division of Developmental Biology at the Roslin Institute, and editor-in-chief of the journal Transgenic Research. Whitelaw also said, "The world must now believe that iPS cells can be truly pluripotent". "Yes, Xiaoxiao received the torch lit by Dolly (the cloned sheep)." Whitelaw commented while reviewing the history of clone.

In later years, scientists at this very institute (IOZ) has also taken the lead in establishing cell lines of mammalian parthenogenetic and androgenetic haploid embryonic stem cells (ESCs), which promises a valuable platform for studying gene function. By modifying the genetic imprints (DNA methylation sites) of the haploid ESCs, they found parthenogenetic and androgenetic haploid ESCs can be functionally transformed into "sperms" and "eggs", respectively, and successfully created live mouse offspring descending from either two egg cells or two sperm cells (Cell Stem Cell 2018. 10.1016/j.stem.2018.09.004). This achievement proclaims the possibility to overcome the restriction of sexual reproduction in mammals.

Later in 2013, scientists at the CAS Guangzhou Institutes of Biomedicine and Health (CIBH) developed a new method to reprogram cells from human urine into immature brain cells that can grow into multifunctional neurons and glial cells (Nature Methods 2013, doi: $10.1038 /$ nmeth.2283). It has opened a new

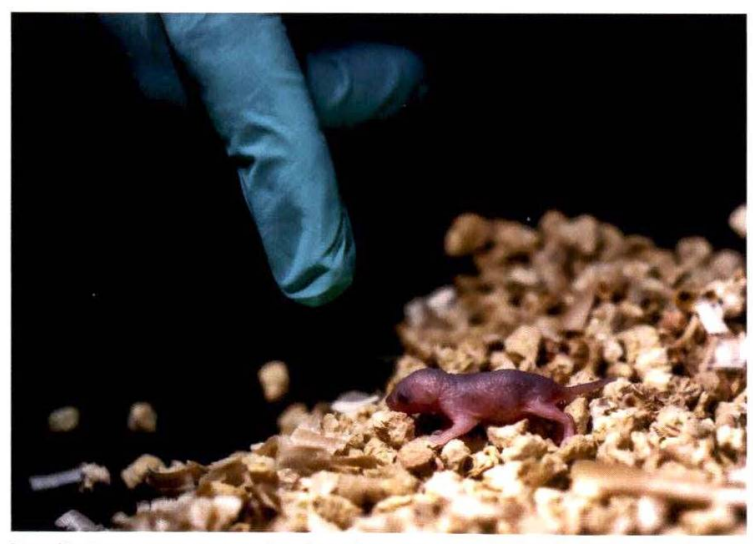

The first mouse pup who has two daddies without a mammy is created in a lab at the Institute of Zoology, CAS. (Credit: WANG Leyun) 


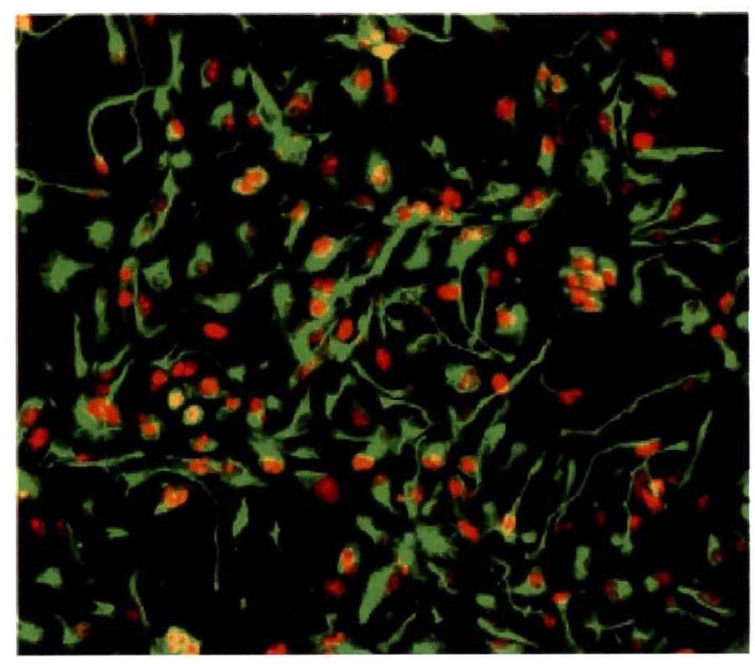

Neuron-related markers of the neural progenitor cells (NPCs) reprogrammed from human urine cells are shown in color. (Credit: Noture Methods)

routine for the treatment of neurological diseases, and could be useful for studying the cellular mechanisms of neurodegenerative conditions and for testing new drugs developed to treat them.

In 2016, scientists at the CAS Center for Excellence in Molecular Cell Science successfully produced hepatocytes from other types of cells. and developed a new type of "biological artificial liver". which has been used to treat and save more than 10 patients with liver failure, and has made its way to industrial transformation.

Besides, scientists at the CAS Institute of Genetics and Developmental Biology (IGDB) have been using spinal nerve-regenerating collagen scaffolds and cell transplantation to treat spinal cord injuries since 2015 . This feat has been rewarded with good results. In the period from 2013 to 2018, ICDB scientists also made breakthroughs in treating endometrial damage and premature ovarian failure by using stem cells combined with collagen scaffolds, which is expected to become an effective treatment for female reproductive diseases.

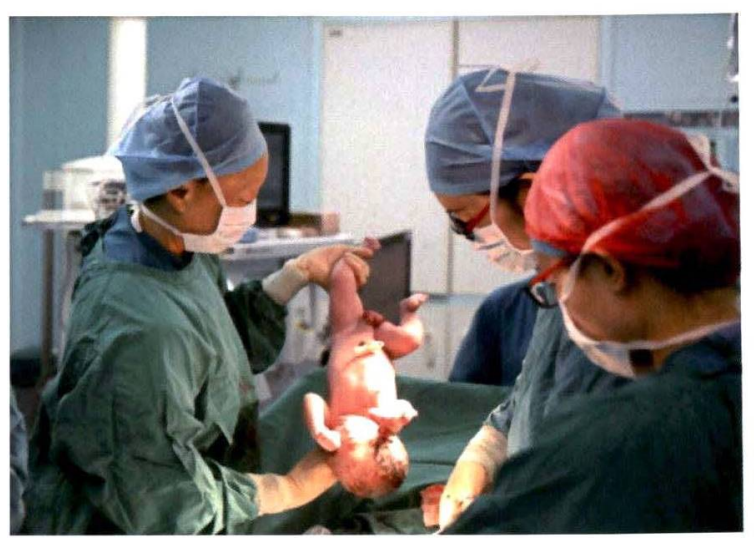

Thanks to the stem cell-based treatment for premature ovarian failure, a Chinese female gives birth to her first healthy baby. (Image by the courtesy of CAS) 